\title{
Convective Transport Suppression in the Scrape-Off Layer Using Ion Cyclotron Resonance Heating on the ASDEX Upgrade Tokamak
}

\author{
G. Antar, ${ }^{1}$ S. Assas, ${ }^{2}$ V. Bobkov, ${ }^{2}$ J.-M. Noterdaeme, ${ }^{2}$ E. Wolfrum, ${ }^{2}$ A. Herrmann, ${ }^{2}$ \\ V. Rohde, ${ }^{2}$ and ASDEX Upgrade Team ${ }^{2}$ \\ ${ }^{1}$ American University of Beirut, Riad el-Solh, Beirut 1107-2020, Lebanon \\ ${ }^{2}$ Max-Planck Institut für Plasmaphysik, Boltzmannstraße 2, 85748 Garching, Germany
}

(Received 15 April 2010; published 12 October 2010)

\begin{abstract}
Turbulence properties in the scrape-off layer (SOL) in the presence of ion cyclotron frequency heating (ICRH) are compared to instances where it is absent. The discharges are all in a high-confinement mode ( $H$-mode) regime. During ICRH, the SOL plasma density increases whereas turbulence large-scale and convective structures are shown to be suppressed. The probability distribution function is thus recorded to be closer to a Gaussian, and a net decrease in the low-frequency density fluctuations is reflected in the power spectra. Consequently, the level of turbulent fluctuations decreases significantly. Turbulence suppression is also reported during edge localized modes (ELMs) where both the ELMs-induced transport and duration are strongly affected. The increase of neutrals by gas puffing did not alter this behavior. We deduce that ICRH can be used as to suppress convective transport and reduce the ELM's amplitude.
\end{abstract}

The effects of the ion cyclotron resonance heating (ICRH) on the scrape-off layer (SOL) has been an outstanding issue for several decades emphasized by the search for efficient coupling of additional heating to the plasma without generating impurities. ICRH effects on the plasma edge were extensively studied on different fusion devices [1-6] in order to understand heat deposition and impurities generation. It is now admitted that part of the power is delivered directly to the SOL leading to a local increase in plasma density and in the impurity levels $[5,7]$. Several mechanisms were investigated, namely, the sheath in front of the antennas where its potential accelerates particles leading to sputtering and a higher impurity concentration. With higher SOL density, more ions are accelerated causing more sputtering. The generation of electric fields in the SOL was observed to be important but not at the edge $[8,9]$. The first study of turbulence behavior in the presence of ICRH was done on the compact helical system where a decrease in the level of fluctuations was observed as the ICRH power exceeded $300 \mathrm{~kW} \mathrm{[10].} \mathrm{On} \mathrm{the} \mathrm{Tore}$ Supra tokamak, local plasma density was shown to be closely related to, among other parameters, the presence of neutrals in the SOL [11]. Recently, it was suggested that convective cells may be forming in front of the antenna [12], which could be in agreement with the theory [13]. On the other hand, intermittency in the SOL was shown to be caused by convective large-scale structures that are called avaloids [14] or blobs [15]. This transport has a universal character with similar properties of turbulence detected on various devices $[16,17]$. Most of the experimental results were done in a low confinement mode ( $L$ mode). Recently, high-confinement mode ( $H$ mode) neutral beam injection (NBI) was found to have the same type of fluctuations in the SOL between edge localized modes (ELMs) as in
$L$ mode where neither the power spectrum nor the probability distribution function were observed to be modified [18] in the $L$ to $H$ transition.

We present experimental results showing that turbulence in the SOL is strongly modified during ICRH with the convective large-scale component suppressed. ELMs are strongly affected where the amount of plasma transported in the SOL is also significantly reduced. During ICRH, midplane gas puffing does not modify this picture.

The main plasma properties of the ASDEX Upgrade Tokamak [19] are shown in Fig. 1 for the two discharges 21408 and 21409. $H$ mode is achieved using $5 \mathrm{MW}$ of NBI leading to type I ELMs seen in the $D_{\alpha}$ time trace [Fig. 1(c)]. The magnetic field is $-2.3 \mathrm{~T}$, the plasma current is $1 \mathrm{MA}$, and the plasma is in the lower-single null magnetic configuration with a major and minor radii, respectively, equal to 1.172 and $0.44 \mathrm{~m}$. The normalized plasma density $n / n_{G}$, where $n_{G}$ is the Greenwald density, is 0.54 dropping to 0.45 in 21408 and to about 0.5 in 21409 during the ICRH [Fig. 1(e)]. Divertor gas valves were used in 21408, whereas in 21409 the target valves were shot down during the ICRH phase and replaced by midplane gas puffing as shown in Fig. 1(g). Consequently, the neutral gas pressure at the midplane is almost unchanged in 21408 whereas it increases sharply in 21409 as illustrated in Fig. 1(d). The four antennas are used with approximately $1 \mathrm{MW}$ on each, leading to $2.7 \mathrm{MW}$ of coupled power. The antennas are set in a dipole configuration where $k_{\|} \simeq 7.5 \mathrm{~m}^{-1}$.

The average density profile between ELMs was measured using the lithium beam diagnostic (LiB) and plotted in Figs. 2(a1) and 2(a2). The increase in the SOL plasma density is small in 21408 but rather large in 21409 where more midplane neutrals were puffed [see Figs. 1(d) and $1(\mathrm{~g})]$. The increase in the SOL density is 


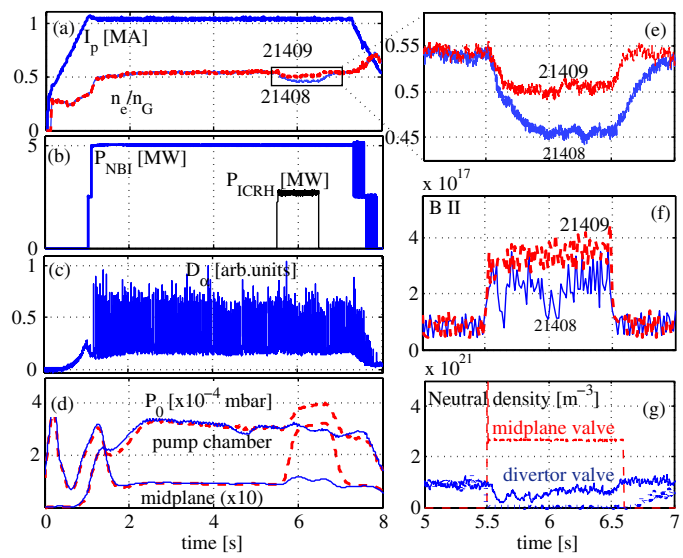

FIG. 1 (color online). Solid lines and dashed lines indicate data taken in 21408 and 21409, respectively. Panel (a) illustrates the plasma current (in MA) and the normalized plasma density; the dip in the core plasma density during ICRH is zoomed on in (e). In (b) we plot the NBI and the coupled ICRH (in MW) power as a function of time. The $D_{\alpha}$ in (c) shows the ELMs, indicating that the discharge remains in $H$ mode. The neutral gas pressure at the divertor and the midplane (multiplied by 10) are shown in (d) where the increase at $5.5 \mathrm{~s}$ in 21409 is caused by the midplane valve's opening shown in $(\mathrm{g})$. The solid lines and the dotted lines in $(\mathrm{g})$ indicate the injected gas density in 21408 and 21409, respectively, from the divertor valves. The dashed line illustrates the gas density injected by midplane valves. The boron II emission line from the antenna shield is plotted in (f) in photons $/\left(\mathrm{m}^{2} \mathrm{srs}\right)$.

also detected by fixed Langmuir probes at the midplane and the target. Their ion saturation current $\left(I_{\text {sat }}\right)$ is shown in Figs. 2(b) and 2(c). As for the impurities' behavior, the boron II emission is illustrated in Fig. 1(f), indicating that the sputtering doubles in 21409 with respect to 21408 . We recall that during ICRH the core density actually decreases [Fig. 1(e)]. One can deduce the following chain: more neutrals leads to more plasma in the SOL and thus more sputtering of the plasma facing components.

Plasma in the SOL is also caused by radial transport across the separatrix and might also explain the increase of

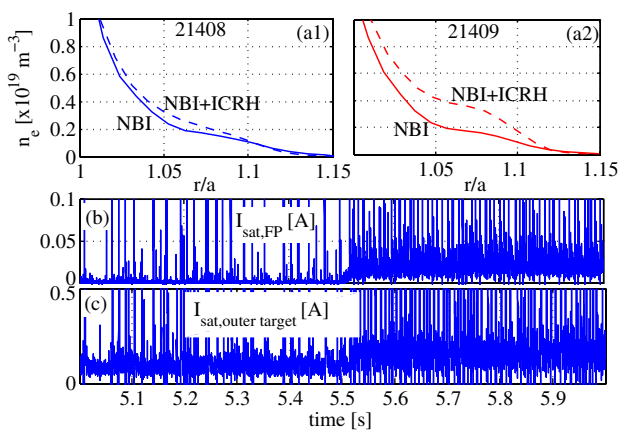

FIG. 2 (color online). Panels (a1) and (a2) illustrate the average density profile from LiB for shots 21408 and 21409. Dashed lines and solid lines illustrate, respectively, data with and without ICRH. In (b) and (c), $I_{\text {sat }}$ is shown for a midplane and a highfield side target probe during 21409. the plasma density. Turbulence is measured using a fixed Langmuir probe biased to the ion saturation current located $40 \mathrm{~cm}$ above the geometrical midplane, about $3 \mathrm{~cm}$ away from the separatrix and $1 \mathrm{~cm}$ in front of the limiter. Figure 3(a) shows the $I_{\text {sat }}$ signals for the shot 21408 before and during ICRH. The contribution of ELMs was removed from the data, causing empty lapses in 3(a). The intermittent bursts clearly present without ICRH are absent with ICRH. This is made even clearer in Fig. 3(b) where the empty time lapses are removed and almost all the spikes which caused SOL intermittency have disappeared.

In order to assess the role of the total additional power, $P_{\text {tot }}$, in affecting the SOL turbulence, we compare the $I_{\text {sat }}$ of discharge 21408 where $P_{\text {tot }}=P_{\mathrm{NBI}}=5 \mathrm{MW}$ to the fluctuations, plotted in Fig. 3(c), obtained by the same probe under similar plasma conditions (shot 20964) but with $P_{\text {tot }}=5.3 \mathrm{MW}$ caused by 2.8 of NBI and 2.5 of ICRH. With about the same total power, intermittency is observed to be suppressed in 20964, when using ICRH, whereas it is not in 21408 where $P_{\mathrm{ICRH}}=0$. Another comparison is also performed between $I_{\text {sat }}$ fluctuations in 21408 where $P_{\text {tot }}=7.7 \mathrm{MW}\left(P_{\mathrm{NBI}}=5, P_{\mathrm{ICRH}}=2.7\right)$ and those taken from discharge 21868 with the same probe and similar plasma conditions but with $P_{\text {tot }}=P_{\mathrm{NBI}}=$ 7.5 MW. The latter are plotted in Fig. 3(d) where, despite
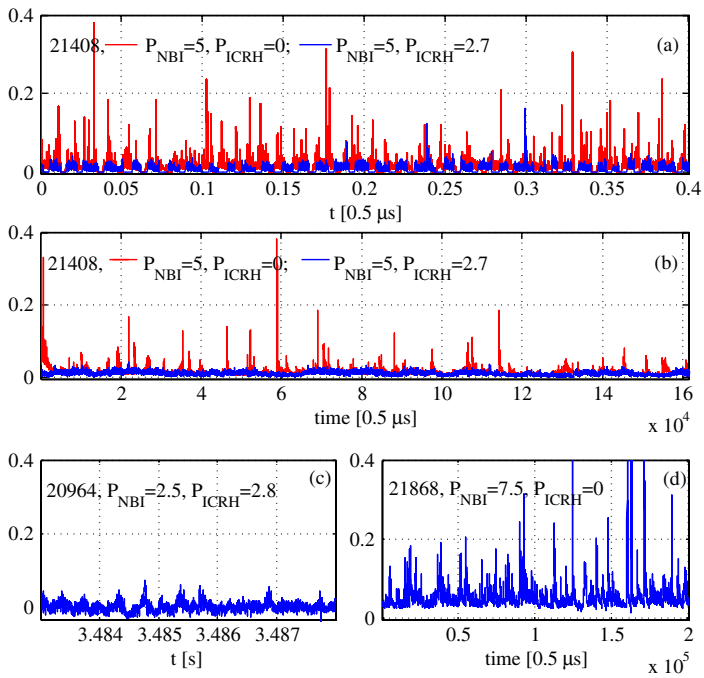

FIG. 3 (color online). In (a), we plot $I_{\text {sat }}$ (in A) of the midplane filament probe with [black (blue)] and without [gray (red)] ICRH. The initial times for the two plots are, respectively, $t_{0} \sim 2$ and $6.1 \mathrm{~s}$. The empty time lapses are mainly caused by ELMs and negative biasing to avoid sparks that we have removed. In (b), we removed the empty time lapses in order to clearly show the radical changes occurring to $I_{\text {sat }}$ when ICRH is switched on. In (c), we inserted $I_{\text {sat }}$ from shot 20964 where $P_{\text {tot }}=5.8 \mathrm{MW}$ coming from 2.5 of NBI and 2.8 of ICRH. The fluctuations can thus be compared to 21408 at $t \sim 2 \mathrm{~s}$ with the same $P_{\text {tot }}$ coming from NBI alone. In (d), the $I_{\text {sat }}$ taken from discharge 21868 with $P_{\text {tot }}=P_{\mathrm{NBI}}=7.5 \mathrm{MW}$ is plotted. Another comparison is thus possible between the fluctuations shown in (d) and those in 21408 around $t \sim 6.1 \mathrm{~s}$ with about the same total power. 
the large additional heating power, intermittency and largescale structures are not suppressed in 21868 whereas they are in 21408 with ICRH. These experimental results clearly indicate that it is the ICRH that is affecting turbulence and not the total power.

In the table below, we insert the average, the standard deviation, and the normalized standard deviation of $I_{\text {sat }}$. The average value increases during ICRH in agreement with the target probe data and the LiB. The level of turbulence, on the other hand, decreases, allowing us to deduce that the main source of the increase of $I_{\text {sat }}$ is not radial transport. Since the average value increases and the standard deviation decreases, it is natural to observe that the relative level of fluctuations decreases rather sharply.

The question now is whether this decrease is accompanied with a change in turbulence properties. It is worth noting that the statistical properties of turbulence in the SOL are not modified in NBI-driven $H$ mode when compared to $L$ mode [18]. We have shown that in the SOL neither the diffusive nor the convective component is modified when NBI is switched on and that the power distribution of the turbulent fluctuations is unmodified.

\begin{tabular}{lllllll} 
Discharge & $P_{\text {ICRH }}(\mathrm{MW})$ & $\left\langle I_{\text {sat }}\right\rangle(\mathrm{mA})$ & $\delta I_{\text {sat }}(\mathrm{mA})$ & $\delta I_{\text {sat }} /\left\langle I_{\text {sat }}\right\rangle$ & Skewness & Flatness \\
\hline 21408 & $0 \rightarrow 2.7$ & $15 \rightarrow 17$ & $10.5 \rightarrow 6$ & $70 \% \rightarrow 35 \%$ & $7.6 \rightarrow 0.8$ & $65 \rightarrow 5.6$ \\
21409 & $0 \rightarrow 2.7$ & $15 \rightarrow 22$ & $10 \rightarrow 7$ & $67 \% \rightarrow 32 \%$ & $12 \rightarrow 1$ & $52 \rightarrow 7$
\end{tabular}

We recall that the plasma remains in $H$ mode during the whole discharge with $5 \mathrm{MW}$ of NBI. We define $I=$ $I_{\text {sat }}-\left\langle I_{\text {sat }}\right\rangle$ where \langle\rangle denotes time average. In Fig. 4(a) the probability distribution functions (PDFs) of $I$ between ELMs are illustrated for plasma with NBI and NBIICRH heating. Negative fluctuations are Gaussian, as it is reflected in the parabolic shape of the PDF [20] and this part remains unmodified when ICRH is added. We deduce that the "diffusive" component of turbulence in the SOL by incoherent eddies is unaltered. The positive values of $I$ were shown to be dominated by the convective component of turbulence caused by large-scale and large radial
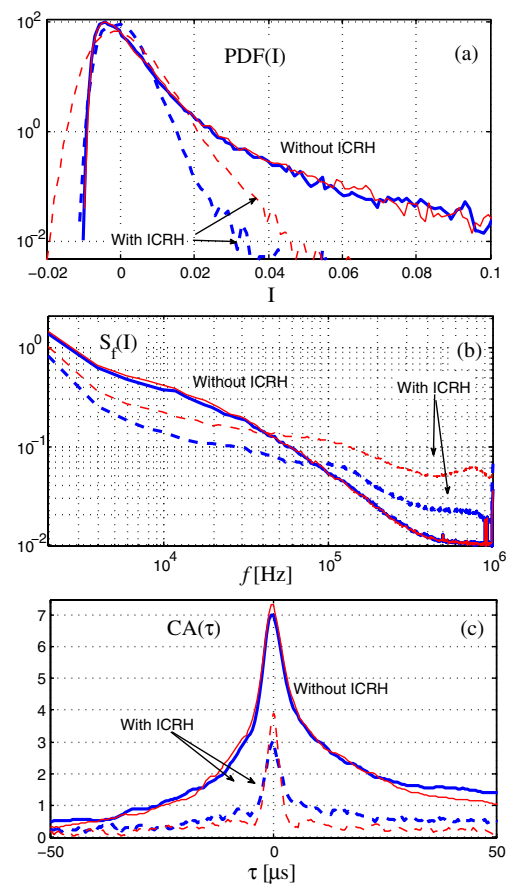

FIG. 4 (color online). In (a), the PDF of $I$ is plotted in a semilogarithmic frame with dashed lines and solid lines for data with and without ICRH, respectively. In (b) and (c), we illustrate the power spectrum of $I$ as a function of the frequency $f$, and the autoconditional average (CA) as a function of time $\tau$ with the same legend for the type of lines as in (a). velocity structures $[14,16]$. This component of the radial transport suffers a dramatic reduction where most of the bursts disappear during ICRH. The change in the PDF shape is quantified using the normalized third and fourth order moments, which are the skewness and the flatness. In 21408 , the skewness drops from 7.6 to 0.8 reflecting a more symmetric PDF and the flatness decreases from 65 to 5.6 approaching 3, the Gaussian value. We deduce that the dramatic change seen visually in the $I_{\text {sat }}$ signals is reflected in the PDF that tends to a Gaussian with the presence of ICRH.

The frequency spectra of $I$ are determined and plotted in Fig. 4(b) where a net decrease in the low-frequency range is reported, whereas the high-frequency part increases slightly. Assuming Taylor's frozen turbulence hypothesis, we deduce that large-scale (small-scale) fluctuations are suppressed (enhanced). This result is in agreement with the PDF behavior and previous results relating the high intensity spikes to large-scale structures. Moreover, we calculated the autoconditional average of the intermittent spikes with and without ICRH heating and plotted the result in Fig. 4(c). The dramatic decrease of the highest amplitude spikes in the signal is clear, reflecting the fact that less plasma is transported across the SOL by the high intensity events. One can also show that the number of large bursts decreases significantly in agreement with the PDF behavior.

In shot 21409 midplane gas puffing is used to avoid the plasma density decrease during ICRH [Fig. 1(e)] leading to core density decreasing from 0.55 to 0.5 and edge density increasing by about $60 \%$ [Figs. 2(a1) and 2(a2)]. The midplane probe indicates that the average value of $I_{\text {sat }}$ between ELMs increases from 17 to $22 \mathrm{~mA}$ while the standard deviation is almost unchanged going from 6 to $7 \mathrm{~mA}$. The statistical properties of turbulence between ELMs can be obtained from Fig. 4. In 4(a), the PDF shows that by increasing the midplane gas puffing, the diffusive transport, reflected mainly in the negative fluctuations part of the PDF, increases as expected [21]. The convective component remains, however, suppressed as the PDF tail for positive I's remains unchanged. This behavior is 


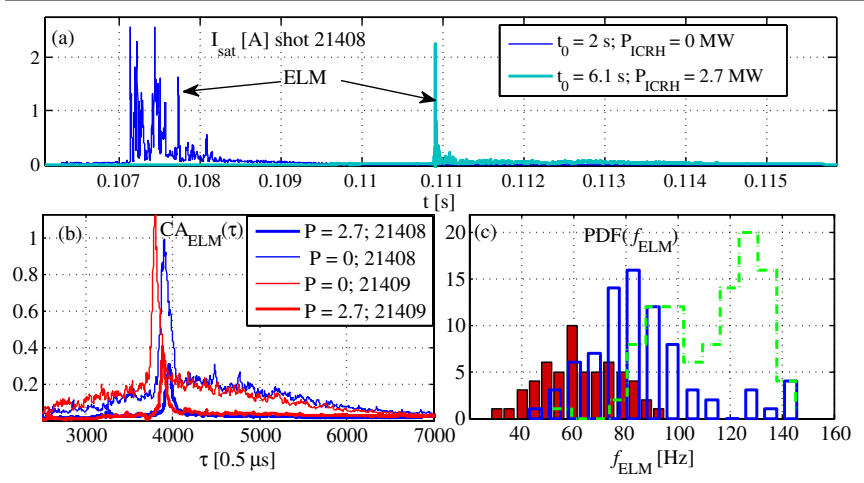

FIG. 5 (color online). In (a) we plot $I_{\text {sat }}$ during 21408 where two ELMs are visible, one with an initial time $t_{0}=2 \mathrm{~s}$ (no ICRH) and the other at $t_{0}=6.1 \mathrm{~s}$ with ICRH. In (b) the autoconditional average of ELMs is illustrated for 21408 and 21409 and with and without ICRH. In (c) the two bar charts reflect the PDF of the ELM frequency $f_{\text {ELM }}$ for 21408 with (filled) and without (empty) ICRH power. The stairs plot shows the same for 21049 with the presence of ICRH.

quantified using the skewness and the flatness factors which remain the same in 21409 when compared to 21408 , indicating that turbulence is not modified. The power spectrum in 4(b) and the autoconditional average in 4(c) give strong indications that turbulence statistical properties are unchanged by the midplane gas puffing. We deduce that with midplane gas puffing the only reported major difference is the increase in the SOL density. The fluctuations' intensities and their statistical properties between ELMs remain unchanged.

ELM-induced transport, on the other hand, is profoundly affected by ICRH, and this is clearly seen on the $I_{\text {sat }}$ plotted in Fig. 5(a) for an arbitrarily chosen ELM with and without ICRH. A net decrease in the signal's amplitude and the duration of the ELM-induced transport are observed. The autoconditional function is determined for ELMs $\left(\mathrm{CA}_{\mathrm{ELM}}\right)$ and plotted in Fig. 5(b) where the above result is verified statistically; that is, the ELM-induced amplitude decreases by about a factor of 3. Comparing 21409 to 21408, we deduce that this decrease is not significantly modified by midplane gas puffing. Accordingly, ICRH suppresses the ELM-induced convective transport similarly to that between ELMs.

The average ELM frequency increases from 60 to $80 \mathrm{~Hz}$ with ICRH in 21408 as shown in Fig. 5(c) in agreement with the linear dependence of ELM frequency on power, which increases from 5 to $7.6 \mathrm{MW}$. Hence, while the amplitude of ELMs decreases sharply (a factor of $1 / 3$ ), only a slight increase in the ELM frequency $(25 \%)$ is observed. In 21409 the ELM frequency doubles, going from 60 to $120 \mathrm{~Hz}$. This is consistent with previous work [22] and in agreement with the linear dependence on power and pedestal density (not central density). In 21409 , the former increases by about $50 \%$ and the latter by about $60 \%$.

We conclude that ICRH leads to (1) turbulence decrease in the SOL via the suppression of large-scale convective structures; this leads to distribution functions close to Gaussian. (2) The ELM-induced transport also is shown to be strongly affected by the ICRH where a decrease by a factor of $1 / 3$ is recorded. (3) Increasing the midplane neutral pressure leads to more density in the SOL but did not affect turbulence suppression. Future work should discuss the physics behind the suppression of the intermittent large-scale structures. Dedicated attention should also be given to ICRH interaction with ELMs and the possibility to control their induced transport by applying power only during their presence.

[1] T. Group, Plasma Phys. Controlled Fusion 26, 1141 (1984).

[2] U. Samm et al., Plasma Phys. Controlled Fusion 29, 1321 (1987).

[3] M. Bures et al., Plasma Phys. Controlled Fusion 30, 149 (1988).

[4] I. S. Lehrman et al., Plasma Phys. Controlled Fusion 32, 51 (1990).

[5] J. V. Hofmann, G. Fussmann, J. M. Noterdaeme, and F. Ryter, Fusion Eng. Des. 12, 185 (1990).

[6] J. M. Noterdaeme and G. V. Oost, Plasma Phys. Controlled Fusion 35, 1481 (1993).

[7] H. Tamai et al., Fusion Eng. Des. 12, 25 (1990).

[8] R. V. Nieuwenhove et al., Fusion Eng. Des. 12, 231 (1990).

[9] R. Van Nieuwenhove et al., Fusion Eng. Des. 12, 231 (1990).

[10] T. Morisaki et al., Plasma Phys. Controlled Fusion 37, 787 (1995).

[11] M. Bécoulet et al., Phys. Plasmas 9, 2619 (2002).

[12] L. Colas et al., Plasma Phys. Controlled Fusion 49, B35 (2007).

[13] J. R. Myra, D. A. D’Ippolito, and M. Bures, Phys. Plasmas 1, 2890 (1994).

[14] G. Y. Antar et al., Phys. Rev. Lett. 87, 065001 (2001).

[15] S. I. Krasheninnikov, Phys. Lett. A 283, 368 (2001).

[16] G. Antar et al., Phys. Plasmas 10, 419 (2003).

[17] B. P. Milligen et al., Phys. Plasmas 12, 052507 (2005).

[18] G. Y. Antar et al., Plasma Phys. Controlled Fusion 50, 095012 (2008).

[19] O. Gruber et al., Nucl. Fusion 41, 1369 (2001).

[20] G. Antar et al., Phys. Plasmas 8, 1612 (2001).

[21] G. Y. Antar et al., Phys. Plasmas 12, 032506 (2005).

[22] J. M. Noterdaeme et al., in Proceedings of the 17th IAEA Conference on Fusion Energy (IAEA CDP/11), Yokohama, Japan, 1998 (IAEA, Yokohama, 1998), pp. F1-CN-69. 